originality of treatment and entire mastery of this intricate and difficult subject. The aim of the authors has been to write a book of moderate size which may be used as the basis of a short course or as a reference book in connection with a lecture course on this subject. The book has many praiseworthy features. Besides being very compact, the reasoning is entirely rigorous and correct, a point which, in view of the lax methods in vogue, cannot be too highly commended. The Borel-Lebesgue theorem on point sets included within intervals or rectangles is employed to establish uniform continuity and similar properties. We note as worthy of mention also the following features : the simple manner of introducing irrational numbers with the corresponding geometrical theory of points on the right line; the methodical use of the upper and lower bounds of a function for simplifying proofs, an extended comparison of functions with regard to magnitude (order, rank) with application to indeterminate forms and very general criteria for the convergence of improper integrals, finally the excellent treatment of integration which develops a surprising amount of the theory in a very small compass.

One who has carefully read this little volume will have acquired not only a goodly supply of facts, but, what is of far greater importance, familiarity with the exact and rigorous reasoning which is a sine qua non of further work in higher analysis.

\title{
James Pierpont.
}

An Algebra for Secondary Schools. By E. R. Hedrick. American Book Company, 1908. $\mathrm{x}+421$ pp.

IT is with pleasure that we welcome this little volume into the already large family of elementary algebras. In these days of rapid changes, when so much that is time honored must make way for the new, there is always room for one more algebra, provided it is written like Professor Hedrick's in a thoroughly modern spirit which seeks to readjust both the material chosen and the manner of its presentation so as to satisfy more nearly the needs of the present day.

The book has many features well calculated to win support among progressive teachers. The style is clear and attractive. The genial personality of the author cannot be swallowed up even in a high school algebra. It pervades the whole book and makes it bright and fresh. Without departing too far 
from established usage, he has succeeded in giving several subjects a new aspect. As far as possible dead wood has been cut out and live material put in its place. The large number of new and attractive problems drawn from everyday life and from the elements of mensuration and physics will be greatly appreciated by the teacher. Considerable prominence has been given to graphical methods. The author has used them very effectively in treating proportion and variation, in the solution of equations, in establishing the notion of roots and logarithms, and even in explaining interpolation in a logarithmic table. Graphical methods not only make these subjects clearer and more real to the pupil, but will prove of great service to him whether he stops study with the high school or not. In the appendix some of the more advanced topics are treated as permutations and combinations, the binomial theorem, limits and infinite series, irrational and complex numbers.

It needs hardly to be added that the reasoning is sound and in accordance with present-day standards. The pupil will have no vicious habits of thought to eradicate if he goes on with his mathematics. In this connection we may note the emphasis the author lays on checking the solution of equations, a point too often entirely overlooked.

It is a good sign for the future of mathematics in this country when men of the calibre of Professor Hedrick are willing to take time from their own researches to write a work of this character. We wish his little book all success.

\section{James Pierpont.}

Die. Lehre von den geometrischen Verwandtschaften. Erster Band: die Verwandtschaften zwischen Gebilden erster Stufe. By Rudolf Sturm. Leipzig and Berlin, B. G. Teubner, 1908. $\mathrm{xii}+415 \mathrm{pp}$.

THE treatise on geometric transformations, of which the present volume is the first part, is estimated to consist of four volumes, the manuscript of which is all in the hands of the printer. The others will be published as soon as possible.

While the first volume is concerned with forms of one dimension, it is not simply a text-book on projective geometry, but considers multiple correspondences, general involutions, trilinearity, and the problem of homography in two and in three dimensions. The first half of the book is concerned with parametric representation, anharmonic ratios, perspectivity, linear 